\title{
Photodissociation of CF3CHO provides a new source of CHF3 (HFC-23) in the atmosphere: implications for new refrigerants.
}

Christopher Hansen ( $\nabla$ christopher.hansen@unsw.edu.au )

School of Chemistry, University of New South Wales, Sydney https://orcid.org/0000-0002-8954-0825 Jyoti Campbell

School of Chemistry, University of New South Wales, Sydney

Scott Kable

School of Chemistry, University of New South Wales, Sydney

Physical Sciences - Article

Keywords: reaction kinetics and dynamics, chlorofluorocarbons (CFCs), hydrochlorofluorocarbons (HCFCs), refrigerants

Posted Date: February 5th, 2021

DOI: https://doi.org/10.21203/rs.3.rs-199769/v1

License: (c) (i) This work is licensed under a Creative Commons Attribution 4.0 International License. Read Full License 


\title{
Photodissociation of $\mathrm{CF}_{3} \mathrm{CHO}$ provides a new source of $\mathrm{CHF}_{3}$ (HFC-23) in the atmosphere: implications for new refrigerants.
}

\author{
Jyoti S. Campbell, ${ }^{1}$ Scott H. Kable, ${ }^{1}$ Christopher S. Hansen ${ }^{1 *}$ \\ ${ }^{1}$ School of Chemistry, University of New South Wales, Sydney NSW 2052, Australia \\ ${ }^{*}$ Correspondence and requests for materials to: christopher.hansen@unsw.edu.au
}

\section{Summary paragraph}

Throughout the last century, chlorofluorocarbons (CFCs) and hydrochlorofluorocarbons (HCFCs) were the most widely used refrigerants. However, it was discovered that they released reactive chlorine in the upper atmosphere causing the hole in the ozone layer and they were phased out by the 1987 Montreal Protocol [1]. They were replaced by hydrofluorocarbons (HFCs), which contain no chlorine and do not damage the ozone layer. However, HFCs are potent greenhouse gases and they were phased out in a 2016 amendment to the Montreal Protocol [2]. The latest evolution of these refrigerants is the hydrofluoroolefins (HFOs). These molecules have no chlorine and incorporate a carbon-carbon double bond to greatly reduce their atmospheric lifetime, and hence their contribution as a greenhouse gas. In this work, we demonstrate that one of the most important HFOs in current use ultimately decomposes partially into HFC-23 $\left(\mathrm{CHF}_{3}\right)$ in the atmosphere. HFC-23 is one of the most potent greenhouse gases known, and the most potent HFC. Despite its phaseout, the observed emissions of HFC-23 have been increasing recently and were the largest in history in 2018 with no conclusive explanation [3]. This work suggests that the production of HFOs might be partially responsible.

\section{Main text}

Halogenated molecules were widely used as refrigerants, propellants, foam blowing agents, fire retardants and other useful chemicals throughout the last century. The chlorofluorocarbons (CFCs) were, by far, the most proliferated until it was realised that their emission was contributing reactive chlorine to the upper atmosphere and causing the hole in the ozone layer. This led to the 1987 Montreal Protocol [1] that governed the global phasedown of CFCs via the hydrochlorofluorocarbons (HCFCs) as interim replacements that still deplete ozone, but to a much lesser extent. Ultimately, CFCs and HCFCs were replaced by hydrofluorocarbons (HFCs), which contain no chlorine and have no ozone depletion potential (ODP). However, HFCs are long-lived and potent greenhouse gases. Global warming potentials (GWPs) are used to compare newlyemitted greenhouse gases according to their contribution to surface heating, typically over 100 years and relative (by mass) to carbon dioxide i.e. $\mathrm{GWP}\left(\mathrm{CO}_{2}\right)=1$. Typical HFCs exhibit GWPs in the thousands and up to 12690 in the case of fluoroform $\left(\mathrm{CHF}_{3}\right.$ or HFC-23) e.g. 1 tonne of fluoroform, emitted today, contributes as much to surface heating over 100 years as 12690 tonnes of $\mathrm{CO}_{2}$. Concerns about offsetting the hard-won environmental gains brought about by the CFC and HCFC phasedown led to the addition of HFCs in the 2016 Kigali amendment to the Montreal Protocol [2].

The leading replacement candidates are a new class of compounds called hydrofluoroolefins (HFOs), which have already been emitted and detected in the atmosphere in significant quantities [4]. These compounds are derived from HFCs but incorporate a carbon-carbon double bond that increases their reactivity, leading to much shorter atmospheric lifetimes (weeks compared to decades/centuries) and near-zero GWPs. One 
important HFO, currently, is HFO-1234ze $\left(\mathrm{CHF}=\mathrm{CH}-\mathrm{CF}_{3}\right)$ with zero ODP and a GWP $<1$ (i.e. less than $\mathrm{CO}_{2}$ ) [5]. These GWP estimates rely on chemical knowledge concerning the decomposition of the HFO and of its onward degradation products. It is known that atmospheric decomposition of HFO-1234ze yields trifluoroacetaldehyde $\left(\mathrm{CF}_{3} \mathrm{CHO}\right)$ with $100 \%$ molar yield [6], which is then removed from the atmosphere $4 \times$ faster than its progenitor via photolysis [7]. Studies dating back to the 1950s suggest that photolysis leads to trifluoromethyl $\left(\mathrm{CF}_{3}\right)$ and formyl $(\mathrm{CHO})$ radicals [8-10] that lead to atmospherically less hazardous $\mathrm{CO}_{2}$ and $\mathrm{HF}[6]$.

The apparently simple photochemistry of $\mathrm{CF}_{3} \mathrm{CHO}$ is in contrast to similar carbonyl species. Acetaldehyde $\left(\mathrm{CH}_{3} \mathrm{CHO}\right)$, the non-fluorinated analogue of $\mathrm{CF}_{3} \mathrm{CHO}$, is known to exhibit far more complex photochemistry than reported for $\mathrm{CF}_{3} \mathrm{CHO}$ with multiple decomposition product channels. Analogous to $\mathrm{CF}_{3} \mathrm{CHO}$, the dominant photolysis pathway for $\mathrm{CH}_{3} \mathrm{CHO}$ at all tropospherically-important wavelengths yields $\mathrm{CH}_{3}$ and $\mathrm{CHO}$ radicals [11-14]. However, following absorption of an ultraviolet photon, $\mathrm{CH}_{3} \mathrm{CHO}$ can also access a rich photochemistry on the ground electronic state $\left(S_{0}\right)$. These many ground state photodissociation pathways are discussed in the following section and, most importantly, include the exoergic formation of methane $\left(\mathrm{CH}_{4}\right)$ and carbon monoxide $(\mathrm{CO})$ [15-17]. Fluoroform $\left(\mathrm{CHF}_{3}\right.$ or HFC-23) is the product analogue of $\mathrm{CH}_{4}$ for $\mathrm{CF}_{3} \mathrm{CHO}$. This is concerning as HFC-23 is the most environmentally hazardous HFC with a GWP of 12 $690[5]$.

In this paper, we demonstrate that $\mathrm{CF}_{3} \mathrm{CHO}$ photolyses to form HFC-23. Although the quantum yield is small, this pathway could account for the fate of $11.0 \pm 5.5 \%$ of atmospheric $\mathrm{CF}_{3} \mathrm{CHO}$. This will considerably increase the 'effective GWP' of any $\mathrm{HFO}$ that includes $\mathrm{CF}_{3} \mathrm{CHO}$ in its decomposition scheme.

\section{Photodissociation pathways}

Fig. 1 presents a potential energy diagram for the various photolysis channels of both $\mathrm{CH}_{3} \mathrm{CHO}$ and $\mathrm{CF}_{3} \mathrm{CHO}$. The minimum energy structures for the intact molecules are shown in the centre and various reaction pathways proceed to the left for $\mathrm{CH}_{3} \mathrm{CHO}$ and to the right for $\mathrm{CF}_{3} \mathrm{CHO}$. The dashed horizontal line represents the energy of a $308 \mathrm{~nm}$ photon $\left(388.4 \mathrm{~kJ} \mathrm{~mol}^{-1}\right)$, which is the wavelength employed in both the current and previous studies [8]. The potential energy schemes are strikingly similar. Several photodissociation channels are energetically open following $308 \mathrm{~nm}$ excitation, and there are no compelling differences that should preclude $\mathrm{CF}_{3} \mathrm{CHO}$ from accessing the same rich ground state photochemistry as its non-fluorinated analogue.

Photolytic cleavage of the $\mathrm{C}-\mathrm{C}$ bond via the well-known Norrish Type-I (NTI) reaction, produces $\mathrm{CH}_{3} / \mathrm{CF}_{3}$ and $\mathrm{CHO}$ (R1, green lines). This reaction is barrierless on the ground electronic state [18], requiring 347 and $335 \mathrm{~kJ} \mathrm{~mol}^{-1}$ for $\mathrm{CH}_{3} \mathrm{CHO}$ and $\mathrm{CF}_{3} \mathrm{CHO}$, respectively. On the lowest energy triplet state, $T_{1}$, the NTI reaction occurs over a small barrier (375 and $367 \mathrm{~kJ} \mathrm{~mol}^{-1}$ respectively). This pathway was not the focus of this study but we carefully studied the dynamics nonetheless since all previous work agrees that it is the dominant photolysis pathway for both species at $308 \mathrm{~nm}$ and it has not yet been explored for $\mathrm{CF}_{3} \mathrm{CHO}$. The details and results of these experiments are in the supplementary information Section $\mathrm{S} 1$ (including Figs. S2 \& S3). The main conclusion is that the dynamics are the same for $\mathrm{CH}_{3} \mathrm{CHO}$ and $\mathrm{CF}_{3} \mathrm{CHO}$ and in accord with the predicted energetics in Fig. 1.

The decarbonylation pathways produce $\mathrm{CO}$, accompanied by $\mathrm{CH}_{4}$ or $\mathrm{CHF}_{3}$ ( $\mathrm{R} 2$, red lines) and are the focus of this study. These are the thermodynamically favoured products, lying 25 and $43 \mathrm{~kJ} \mathrm{~mol}^{-1}$ respectively below their precursors, but proceeding over high barriers of 347 and $325 \mathrm{~kJ} \mathrm{~mol}^{-1}$, respectively. These pathways were investigated using resonance-enhanced multiphoton ionisation (REMPI) with velocitymapped ion imaging (VMI) to probe CO photoproducts with quantum state specificity. We focus on the $\mathrm{CO}$ fragment because VMI depends on the target being ionised for detection, and $\mathrm{CHF}_{3}^{+}$is unstable and decomposes to $\mathrm{CF}_{3}^{+}$[19], which is already observed due to the radical channel (R1). These results are presented and discussed throughout the remaining sections of this article.

The most apparent difference in the photochemistry of these molecules is in the minor pathway producing $\mathrm{CH}_{2} \mathrm{CO}+\mathrm{H}_{2}$ in $\mathrm{CH}_{3} \mathrm{CHO}$ [20]. The transition state for the analogous pathway in $\mathrm{CF}_{3} \mathrm{CHO}$ could not be located; instead a hydrogen-bonding interaction is formed between the formyl $\mathrm{H}$-atom and a fluoromethyl 
fluorine atom leading to $\mathrm{CF}_{2}, \mathrm{HF}$ and $\mathrm{CO}$ products. This predicted pathway has not been reported previously and has no analogue in the photochemistry of $\mathrm{CH}_{3} \mathrm{CHO}$, but is a (potential) competing source of $\mathrm{CO}$ photoproducts and needed to be investigated. We searched for this channel using carefully designed lowpressure Fourier-transform Infrared (FT-IR) experiments with in situ photolysis and found no evidence for it with a detection limit corresponding to a maximum possible quantum yield of $<0.07 \%$. These experiments and analyses are described in detail in Section S2 (including Figs. S4, S5 \& S6) of the supplementary information.

\section{Dynamics of the decarbonylation channel (R2)}

Fig. 2 summarises the key experimental results from this study. Fig. 2A shows the 2D REMPI spectrum of $\mathrm{CO}$ formed from photodissociation of $\mathrm{CF}_{3} \mathrm{CHO}$ at $308 \mathrm{~nm}$. The abscissa records the wavenumber of the $\mathrm{CO}$ detection laser on the lower axis and equivalent $\mathrm{CO}$ rotational quantum number, $J$, on the upper axis, and the ordinate shows the speed of the nascent CO. Two components are evident. The first appears in the lower left of Fig. 2A, outlined by the yellow triangle, and arises from slow $\mathrm{CO}$ with a narrow distribution of low- $J$ states and is the dominant channel. The second component spans the entirety of Fig. $2 \mathrm{~A}$ and reveals CO photoproducts with a broad distribution of speed and $J$. The 2D data in Fig. 2A can be integrated over either speed or wavelength as shown in Figs. 2B and 2C, respectively. Fig. 2B shows the 2D data integrated over low-speed (within the yellow triangle), high-speed and all-speed CO. Fig. 2C shows the orthogonal integration for low- $J$ (within the yellow triangle), high- $J$ and all- $J$. The data in both panels accentuate the bimodality in $\mathrm{CO}$ dynamics with low speed correlated with low $J$ and high speed with high $J$.

This 2D REMPI spectrum is strikingly similar to that published previously for $\mathrm{CH}_{3} \mathrm{CHO}$ [15]. The dissociation of $\mathrm{CH}_{3} \mathrm{CHO}$ via $\mathrm{R} 2$ has been reported to occur via up to three different dynamical channels. Although the interpretation of each channel is still not agreed upon in the literature [15-17], the key factor for this work is that all hypothesised channels yield $\mathrm{CH}_{4}$ and $\mathrm{CO}$ as photoproducts following dynamics on $S_{0}$ after initial photo-excitation to $\mathrm{S}_{1}$. The two different $\mathrm{CO}$ dynamical signatures from $\mathrm{CF}_{3} \mathrm{CHO}$ dissociation in Fig. 2 are consistent with the multi-dynamical processes in $\mathrm{CH}_{3} \mathrm{CHO}$.

\section{Quantum yield of the decarbonylation channel (R2)}

From the experiments above, we conclude that R1 and R2 are active unimolecular photochemical decomposition pathways of $\mathrm{CF}_{3} \mathrm{CHO}$ at $308 \mathrm{~nm}$ while there is no evidence for R3. We take advantage of the similar photochemistry of $\mathrm{CF}_{3} \mathrm{CHO}$ and $\mathrm{CH}_{3} \mathrm{CHO}$, as demonstrated above, and the well-known photochemistry of the latter to determine the quantum yield (QY) of $\mathrm{R} 2$ under low pressure conditions. We utilise $\mathrm{CH}_{3} \mathrm{CHO}$ as an internal standard with which we benchmark $\mathrm{CF}_{3} \mathrm{CHO}$. Three gas cylinders containing pure $\mathrm{He}$, and either $2 \% \mathrm{CF}_{3} \mathrm{CHO}$ or $\mathrm{CH}_{3} \mathrm{CHO}$ pre-mixed in He were prepared in a parallel gas manifold and placed immediately before the pulsed nozzle of the REMPI experiment described above. These gas mixtures could be switched in seconds while all other experimental parameters remained stable.

Fig. 3 shows the relative measurements of nascent $\mathrm{CO}(J=8)$ signal from photolysis of each aldehyde as a function of time. Firstly, the $\mathrm{CF}_{3} \mathrm{CHO}$ supply was switched on for 10 seconds and off for 10 seconds for 10 cycles. The feed gas was then switched to helium, showing the slightly lower background and verifying the removal of residual $\mathrm{CF}_{3} \mathrm{CHO}$. Finally, the feed gas was switched to $\mathrm{CH}_{3} \mathrm{CHO}$ and a similar profile and reproducibility was observed. The relative intensity between $\mathrm{CF}_{3} \mathrm{CHO}$ and $\mathrm{CH}_{3} \mathrm{CHO}$ signal in Fig. 3, the absorption spectrum of each compound [21] and survey CO photoproduct REMPI spectra for each compound (Fig. S7 of the supplementary information) were then used to determine that the nascent (zero-pressure) quantum yield of $\mathrm{R} 2$ from $\mathrm{CF}_{3} \mathrm{CHO}$ at $308 \mathrm{~nm}$ is $2.5 \pm 0.7$ times that of $\mathrm{CH}_{3} \mathrm{CHO}$. Further details about these calculations are in the supplementary information section S3. 


\section{Atmospheric fate of $\mathrm{CF}_{3} \mathrm{CHO}$}

The results above demonstrate unambiguously that $\mathrm{CHF}_{3}+\mathrm{CO}$ is a primary product channel from the photolysis of $\mathrm{CF}_{3} \mathrm{CHO}$ at $308 \mathrm{~nm}$ and that $\mathrm{CF}_{3} \mathrm{CHO}$ and $\mathrm{CH}_{3} \mathrm{CHO}$ exhibit analogous photochemistry. We utilise this to contextualise our results and to estimate the yield of $\mathrm{CHF}_{3}$ under atmospheric conditions. Fig. 4 shows a summary of all previous measurements of the $\mathrm{CH}_{3} \mathrm{CHO}$ and $\mathrm{CF}_{3} \mathrm{CHO}$ quantum yields in air at atmospheric pressure. The most extensive data set for $\mathrm{CH}_{3} \mathrm{CHO}$ was published by Moortgat and co-workers (MMW and WM) [13,14], while earlier measurements by Calvert and co-workers (HC and HKC) [11,12] are in good agreement. Panel A shows total photolysis QY, $\Phi($ tot $)$, as a function of wavelength, while Panels B and C show $\Phi(\mathrm{R} 1)$ and $\Phi(\mathrm{R} 2)$ (i.e. the photolysis QYs for channels R1 and R2) separately.

The function fit through $\Phi($ tot $)$ is the wavelength-pressure dependent quantum yield for $\mathrm{CH}_{3} \mathrm{CHO}$ photodissociation recommended by IUPAC, calculated at atmospheric pressure [22]. There are no recommended functions for the individual quantum yields. IUPAC recommends $\Phi(\mathrm{R} 2)=0$ for $\lambda>300 \mathrm{~nm}$. However, the data in the inset to Fig. 4C show that, although the measured quantum yield at atmospheric pressure is small, it is not zero. The data shown here are consistent with the original publications $[13,14]$, where the authors determine an upper limit of $\Phi(\mathrm{R} 2)<0.005$. Although this small quantum yield has negligible implications for the atmospheric chemistry of $\mathrm{CH}_{3} \mathrm{CHO}$ (which possibly led to the IUPAC recommendation), we argue below that this is not the case for $\mathrm{CF}_{3} \mathrm{CHO}$.

We fit a sigmoidal function to $\Phi(\mathrm{R} 2)$ in Fig. $4 \mathrm{C}$, while the line for $\Phi(\mathrm{R} 1)$ in Fig. $4 \mathrm{~B}$ is the difference between $\Phi($ tot $)$ and $\Phi(\mathrm{R} 2)$. We do not attribute any meaning to the functional form and the important aspect is the non-zero offset at $\lambda>300 \mathrm{~nm}$, which is almost flat. Our sigmoidal fit places this at $\Phi(\mathrm{R} 2)=$ $0.004 \pm 0.002$.

Previous studies of the photochemistry of $\mathrm{CF}_{3} \mathrm{CHO}$ are scarce. The available wavelength-dependent QYs for $\mathrm{CF}_{3} \mathrm{CHO}$ are also shown in Fig. $4[8,9]$. We have demonstrated that the energetics and dynamics of these two carbonyl reactions are remarkably similar. Fig. 4 shows that this is translated through to equally similar QYs under atmospheric conditions. Fig. 4C includes an estimate of $\Phi(\mathrm{R} 2)$ at $308 \mathrm{~nm}$ from this work. Here, we assume the collision-free measurement, $\Phi\left(\mathrm{R} 2, \mathrm{CF}_{3} \mathrm{CHO}\right)=2.5 \times \Phi\left(\mathrm{R} 2, \mathrm{CH}_{3} \mathrm{CHO}\right)$, also applies at atmospheric pressure, i.e. that the quenching kinetics for $\mathrm{CH}_{3} \mathrm{CHO}$ and $\mathrm{CF}_{3} \mathrm{CHO}$ are similar. This provides an estimate of $\Phi(\mathrm{R} 2)=0.010 \pm 0.005$. The previous estimate of $\Phi(\mathrm{R} 2)=0.021$ at $313 \mathrm{~nm}$ [9] lies a little above our estimate, as shown, but was recorded at lower pressure (700 Torr), with correspondingly less quenching and is therefore likely consistent with the present measurement.

The photolysis rate coefficient, $k_{\mathrm{ph}}$, can be calculated as $k_{\mathrm{ph}}=\sum_{\lambda} j(\lambda) \sigma(\lambda) \Phi(\lambda)$ where $j(\lambda)$ is the the actinic flux, $\sigma(\lambda)$ the absorption cross section and $\Phi(\lambda)$ the photolysis quantum yield. Using the actinic flux for an overhead sun on a clear day from the US EPA [23], the published absorption spectrum of $\mathrm{CF}_{3} \mathrm{CHO}$ [10] and the quantum yields from Fig. 4 evaluates to $k_{\mathrm{ph}}(\mathrm{tot})=5.9 \times 10^{-6} \mathrm{~s}^{-1}$ and $k_{\mathrm{ph}}(\mathrm{R} 2)=(7 \pm 4) \times 10^{-7}$ $\mathrm{s}^{-1}$. $\mathrm{CF}_{3} \mathrm{CHO}$ can also decompose in the atmosphere by reaction with $\mathrm{OH}$ with a reported rate coefficient, $k_{\mathrm{OH}}=(4.8 \pm 0.3) \times 10^{-13} \mathrm{~cm}^{3}$ molecules ${ }^{-1} \mathrm{~s}^{-1}[10]$. Using the global average concentration of $[\mathrm{OH}]=1.1$ $\times 10^{6}$ molecules $\mathrm{cm}^{-3}[24]$ yields a pseudo-first order rate coefficient for reaction with $\mathrm{OH}$ of $k_{\mathrm{OH}}^{\prime}=5.3 \times$ $10^{-7} \mathrm{~s}^{-1}$. These rate coefficients govern the atmospheric fate of $\mathrm{CF}_{3} \mathrm{CHO}$ resulting in a half-life of $\sim 4$ days with the dominant radical channel (R1) accounting for the removal of $79 \%$ of atmospheric $\mathrm{CF}_{3} \mathrm{CHO}_{1} 10 \%$ decomposing by reaction with $\mathrm{OH}$ and $11 \%$ of $\mathrm{CF}_{3} \mathrm{CHO}$ in the atmosphere undergoing the decarbonylation reaction, R2, leading to $\mathrm{CO}$ and $\mathrm{CHF}_{3}$ (HFC-23). Details of the calculations can be found in supplementary information Section S4, including Tables S1 \& S2.

The photochemistry of $\mathrm{CH}_{3} \mathrm{CHO}$ and $\mathrm{CF}_{3} \mathrm{CHO}$ are fundamentally the same, however, the $\mathrm{CH}_{4}$ pathway in $\mathrm{CH}_{3} \mathrm{CHO}$ comprises only about $1 \%$ of the atmospheric fate, in comparison to the $11 \%$ calculated above for HFC-23 from $\mathrm{CF}_{3} \mathrm{CHO}$. The underlying difference is that the $\mathrm{CH}_{3} \mathrm{CHO}$ reaction with $\mathrm{OH}$ has a rate coefficient $\left(k_{\mathrm{OH}}=1.5 \times 10^{-11} \mathrm{~s}^{-1}[22]\right)$ that is over an order of magnitude greater than that for $\mathrm{CF}_{3} \mathrm{CHO}$. While $\mathrm{CF}_{3} \mathrm{CHO}$ undergoes an average of 11 absorption cycles before reacting with $\mathrm{OH}$ and a $1 \%$ quantum yield becomes an $11 \%$ reaction fate, $\mathrm{CH}_{3} \mathrm{CHO}$ absorbs only $\sim 2$ photons on average before reacting. 


\section{Implications for $\mathrm{HFO}$ use}

We have determined that $\mathrm{CHF}_{3}+\mathrm{CO}$ is unambiguously a primary photochemical product channel of $\mathrm{CF}_{3} \mathrm{CHO}$. Under collision-free conditions, the quantum yield is 2.5 times higher than for the equivalent channel in $\mathrm{CH}_{3} \mathrm{CHO}$. The dynamics, energetics and $\mathrm{QYs}$ of $\mathrm{CF}_{3} \mathrm{CHO}$ are very similar to those of $\mathrm{CH}_{3} \mathrm{CHO}$. Utilising the more extensive data for $\mathrm{CH}_{3} \mathrm{CHO}$ and the measurements in this work for $\mathrm{CF}_{3} \mathrm{CHO}$, we estimate the quantum yield of $\mathrm{CHF}_{3}$ under atmospheric conditions to be $(1.0 \pm 0.5) \%$. Because the rate coefficient for reaction with $\mathrm{OH}$ is very slow, this $1 \%$ quantum yield results in $(11.0 \pm 5.5) \%$ of $\mathrm{CF}_{3} \mathrm{CHO}$ molecules in the atmosphere decomposing via this $\mathrm{CHF}_{3}$ pathway, resulting in a secondary GWP $=1400 \pm 700$ for $\mathrm{CF}_{3} \mathrm{CHO}$.

$\mathrm{CF}_{3} \mathrm{CHO}$ is formed in $100 \%$ molar yield from the atmospheric decomposition of HFO-1234ze [6] as well as from the decomposition of other HFOs [25] and HFCs [26]. This introduces a concern that HFOs being considered as replacement gases for air conditioning and other industrial uses might have significant impact as climate change agents. We note that if the GWP for $\mathrm{CF}_{3} \mathrm{CHO}$ from this work withstands further scrutiny, the European Union would re-classify HFO-1234ze from a 'low or no GWP' compound into one that is forbidden [27].

At this stage, we refrain from recommending the withdrawal of HFO-1234ze from commercial use; there are too many uncertainties at present. The most significant uncertainty is using quantum yields from $\mathrm{CH}_{3} \mathrm{CHO}$ as a proxy for $\mathrm{CF}_{3} \mathrm{CHO}$, despite the similarity shown in Fig. 4. More research is needed measuring the wavelength and pressure dependence of quantum yields for $\mathrm{R} 1$ and $\mathrm{R} 2$ for $\mathrm{CF}_{3} \mathrm{CHO}$ explicitly and we call for urgent experiments in this regard.

In closing, we note that atmospheric monitoring has revealed an unknown source of HFC-23 over the past 3 years [3]. After decreasing for several years, the emission of $\mathrm{CHF}_{3}$ in 2018 was higher than measured any year in history. The authors of that work explicitly suggest that China might not have met abatement targets. However, this study could provide an alternative explanation whereby legal production of HFOs might produce HFC-23 as a secondary atmospheric product thereby accounting for some of the observed increase.

\section{References}

[1] United Nations Environment Programme. Montreal Protocol on Substances that Deplete the Ozone Layer (Montreal, 15 September 1987), 1987.

[2] United Nations Environment Programme. Amendment to the Montreal Protocol on Substances that Deplete the Ozone Layer (Kigali, 15 October 2016), 2016.

[3] K. M. Stanley, D. Say, J. Muhle, C. M. Harth, P. B. Krummel, D. Young, S. J. O'Doherty, P. K. Salameh, P. G. Simmonds, R. F. Weiss, R. G. Prinn, P. J. Fraser, and M. Rigby. Increase in global emissions of HFC-23 despite near-total expected reductions. Nature Communications, 11(1), 2020.

[4] M. K. Vollmer, S. Reimann, M. Hill, and D. Brunner. First Observations of the Fourth Generation Synthetic Halocarbons HFC-1234yf, HFC-1234ze(E), and HCFC-1233zd(E) in the Atmosphere. Environmental Science \& Technology, 49(5):2703-2708, 2015.

[5] World Meteorological Organization Global Ozone Research and Monitoring Project. Report No. 58: Scientific Assessment of Ozone Depletion: 2018, 2018.

[6] M. S. Javadi, R. Sondergaard, O. J. Nielsen, M. D. Hurley, and T. J. Wallington. Atmospheric chemistry of trans- $\mathrm{CF}_{3} \mathrm{CH}=\mathrm{CHF}$ : products and mechanisms of hydroxyl radical and chlorine atom initiated oxidation. Atmospheric Chemistry and Physics, 8(12):3141-3147, 2008.

[7] R. Atkinson, D. L. Baulch, R. A. Cox, J. N. Crowley, R. F. Hampson, R. G. Hynes, M. E. Jenkin, M. J. Rossi, J. Troe, and T. J. Wallington. Evaluated kinetic and photochemical data for atmospheric 
chemistry: Volume IV - gas phase reactions of organic halogen species. Atmospheric Chemistry and Physics, 8(15):4141-4496, 2008.

[8] M. S. Chiappero, F. E. Malanca, G. A. Arguello, S. T. Wooldridge, M. D. Hurley, J. C. Ball, T. J. Wallington, R. L. Waterland, and R. C. Buck. Atmospheric chemistry of perfluoroaldehydes $\left(\mathrm{C}_{x} \mathrm{~F}_{2 x+1} \mathrm{CHO}\right)$ and fluorotelomer aldehydes $\left(\mathrm{C}_{x} \mathrm{~F}_{2 x+1} \mathrm{CH}_{2} \mathrm{CHO}\right)$ : Quantification of the important role of photolysis. Journal of Physical Chemistry A, 110(43):11944-11953, 2006.

[9] R. E. Dodd and J. W. Smith. The Photolysis of Trifluoroacetaldehyde. Journal of the Chemical Society, pages 1465-1473, 1957.

[10] S. R. Sellevag, T. Kelly, H. Sidebottom, and C. J. Nielsen. A study of the IR and UV-Vis absorption cross-sections, photolysis and $\mathrm{OH}$-initiated oxidation of $\mathrm{CF}_{3} \mathrm{CHO}$ and $\mathrm{CF}_{3} \mathrm{CH}_{2} \mathrm{CHO}$. Physical Chemistry Chemical Physics, 6(6):1243-1252, 2004.

[11] A. Horowitz and J. G. Calvert. Wavelength dependence of the primary processes in acetaldehyde photolysis. Journal of Physical Chemistry, 86(16):3105-3114, 1982.

[12] A. Horowitz, C. J. Kershner, and J. G. Calvert. Primary Processes in the photolysis of acetaldehyde at $3000 \AA$ and $25^{\circ} \mathrm{C}$. Journal of Physical Chemistry, 86(16):3094-3105, 1982.

[13] G. K. Moortgat, H. Meyrahn, and P. Warneck. Photolysis of Acetaldehyde in Air: $\mathrm{CH}_{4}, \mathrm{CO}$ and $\mathrm{CO}_{2}$ Quantum Yields. ChemPhysChem, 11(18, SI):3896-3908, 2010.

[14] P. Warneck and G. K. Moortgat. Quantum yields and photodissociation coefficients of acetaldehyde in the troposphere. Atmospheric Environment, 62:153-163, 2012.

[15] K. L. K. Lee, M. S. Quinn, A. T. Maccarone, K. Nauta, P. L. Houston, S. A. Reid, M. J. T. Jordan, and S. H. Kable. Two roaming pathways in the photolysis of $\mathrm{CH}_{3} \mathrm{CHO}$ between 328 and $308 \mathrm{~nm}$. Chemical Science, 5(12):4633-4638, 2014.

[16] H.-K. Li, P.-Y. Tsai, K.-C. Hung, T. Kasai, and K.-C. Lin. Communication: Photodissociation of $\mathrm{CH}_{3} \mathrm{CHO}$ at $308 \mathrm{~nm}$ : Observation of H-roaming, $\mathrm{CH}_{3}$-roaming, and transition state pathways together along the ground state surface. Journal of Chemical Physics, 142(4), 2015.

[17] C.-H. Yang, S. Bhattacharyya, L. Liu, W.-H. Fang, and K. Liu. Real-time tracking of the entangled pathways in the multichannel photodissociation of acetaldehyde. Chemical Science, 11(25):6423-6430, 2020.

[18] B. R. Heazlewood, A. T. Maccarone, D. U. Andrews, D. L. Osborn, L. B. Harding, S. J. Klippenstein, M. J. T. Jordan, and S. H. Kable. Near-threshold H/D exchange in $\mathrm{CD}_{3} \mathrm{CHO}$ photodissociation. Nature Chemistry, 3(6):443-448, 2011.

[19] F. C.-Y. Wang and G. E. Leroi. Photoionization and fragmentation of halogenated methanes. Annals of the Israel Physical Society, 6:210-214, 1983.

[20] A. W. Harrison, A. Kharazmi, M. F. Shaw, M. S. Quinn, K. L. K. Lee, K. Nauta, K. N. Rowell, M. J. T. Jordan, and S. H. Kable. Dynamics and quantum yields of $\mathrm{H}_{2}+\mathrm{CH}_{2} \mathrm{CO}$ as a primary photolysis channel in $\mathrm{CH}_{3}$ CHO. Physical Chemistry Chemical Physics, 21(26):14284-14295, 2019.

[21] H. Keller-Rudek, G. K. Moortgat, R. Sander, and R. Soerensen. The MPI-Mainz UV/VIS Spectral Atlas of Gaseous Molecules of Atmospheric Interest. Earth System Science Data, 5(2):365-373, 2013.

[22] R. Atkinson, D. L. Baulch, R. A. Cox, J. N. Crowley, R. F. Hampson, R. G. Hynes, M. E. Jenkin, M. J. Rossi, and J. Troe. Evaluated kinetic and photochemical data for atmospheric chemistry: Volume II gas phase reactions of organic species. Atmospheric Chemistry and Physics, 6:3625-4055, 2006. 
[23] Peterson, J. T. Document EPA-600/4-76-025. Calculated Actinic Fluxes (290 - 700 nm) for Air Pollution Photochemistry Applications. US EPA, 1976.

[24] M. Li, E. Karu, C. Brenninkmeijer, H. Fischer, J. Lelieveld, and J. Williams. Tropospheric OH and stratospheric $\mathrm{OH}$ and $\mathrm{Cl}$ concentrations determined from $\mathrm{CH}_{4}, \mathrm{CH}_{3} \mathrm{Cl}$, and $\mathrm{SF}_{6}$ measurements. npj Climate and Atmospheric Science, 1, 2018.

[25] P. K. Rao and S. P. Gejji. Atmospheric degradation of HCFO-1233zd(E) initiated by OH radical, Cl atom and $\mathrm{O}_{3}$ molecule: Kinetics, reaction mechanisms and implications. Journal of Fluorine Chemistry, 211:180-193, 2018.

[26] European Centre for Ecotoxicology and Toxicology of Chemicals. Joint Assessment of Commodity Chemicals report 44 - 1,1,1,3,3-Pentafluoropropane (HFC-245FA), 2004.

[27] Official Journal of the European Union. Directive 2006/40/EC of the European Parliament and of the Council of 17 May 2006 relating to emissions from air conditioning systems in motor vehicles and amending Council Directive 70/156/EEC, 2006.

[28] B. Chan, J. Deng, and L. Radom. G4(MP2)-6X: A Cost-Effective Improvement to G4(MP2). Journal of Chemical Theory and Computation, 7(1):112-120, 2011.

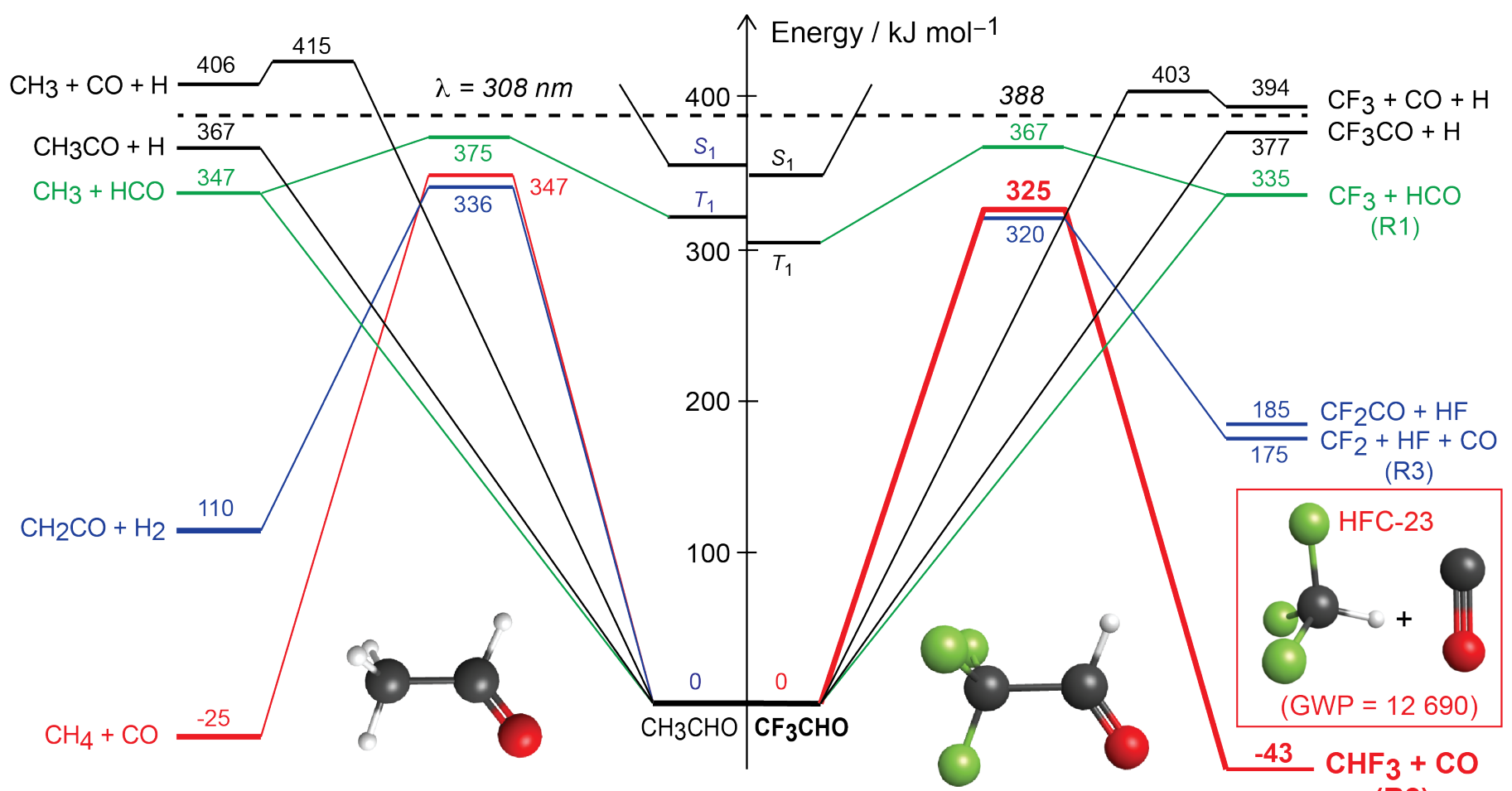

(R2)

Figure 1: Potential energy scheme for the photodissociation of $\mathrm{CF}_{3} \mathrm{CHO}$ and $\mathrm{CH}_{3} \mathrm{CHO}$. The $\mathrm{CH}_{3} \mathrm{CHO}$ energies are experimental values from the references cited in the text and the $\mathrm{CF}_{3} \mathrm{CHO}$ energies were calculated using the G4(MP2)-6x method [28]. The product and transition state energies are relative to the groundstate energy of the equilibrium geometry of each respective species. The dashed horizontal line represents the energy of a photon at $308 \mathrm{~nm}$. 


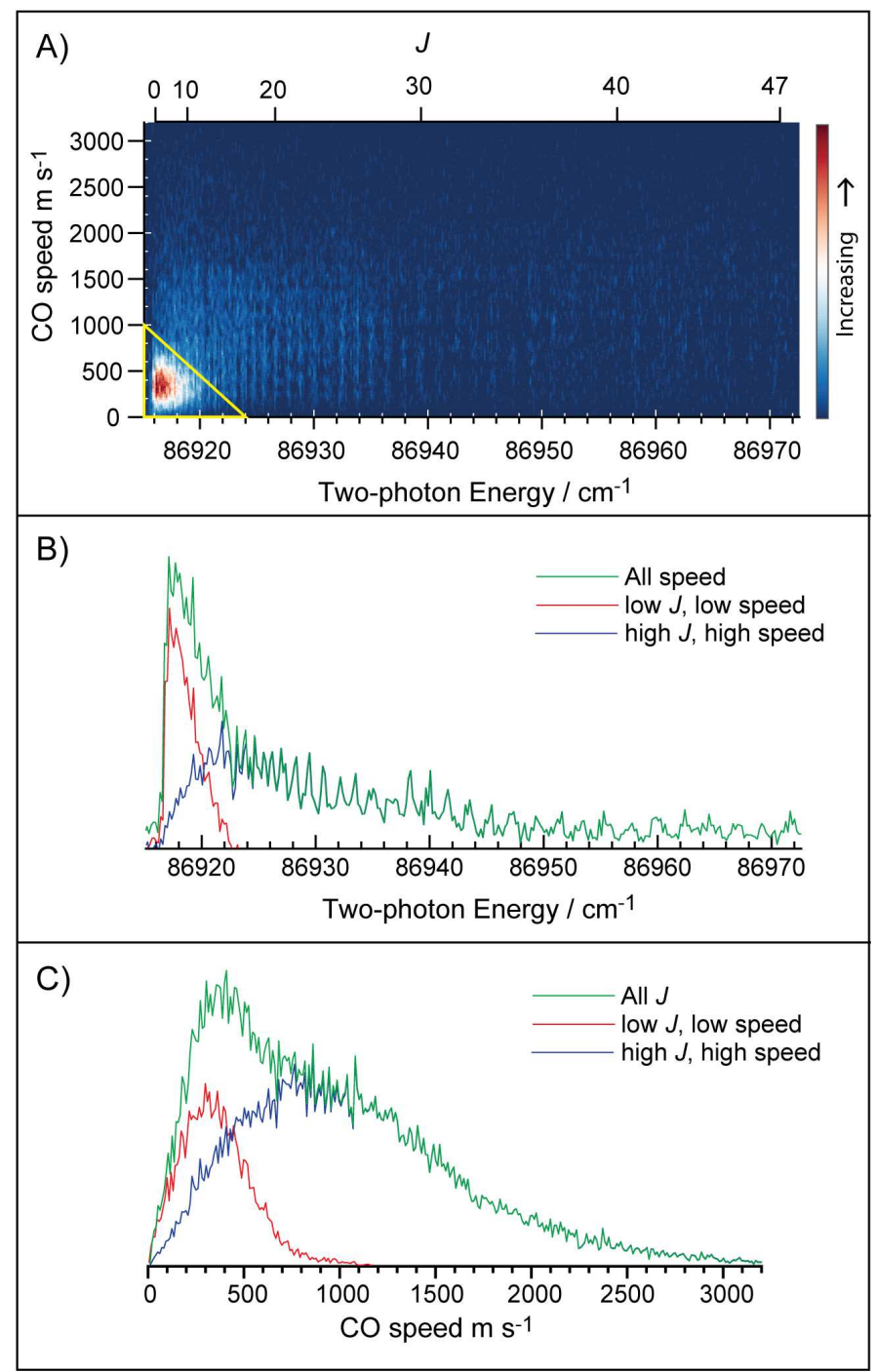

Figure 2: (A) 2D REMPI spectrum of the $\mathrm{CO}$ fragments formed following photolysis of $\mathrm{CF}_{3} \mathrm{CHO}$ at 308 nm. B) CO REMPI spectra from integrating (A) at low speed (within the yellow triangle, red), high speed (outside the yellow triangle, blue) and all speeds (green). C) Speed distributions determined by integrating (A) within and without the yellow triangle and across all $J$. 


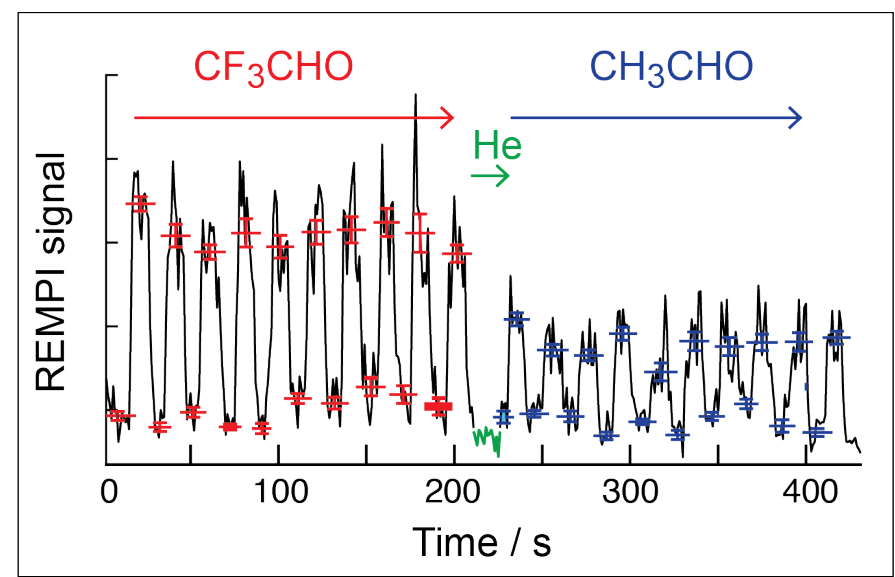

Figure 3: Time-dependent $\mathrm{CO}(J=8)$ ion signal as the molecular beam is switched on and off and from $\mathrm{CF}_{3} \mathrm{CHO}$ to $\mathrm{CH}_{3} \mathrm{CHO}$ as described in the text. 


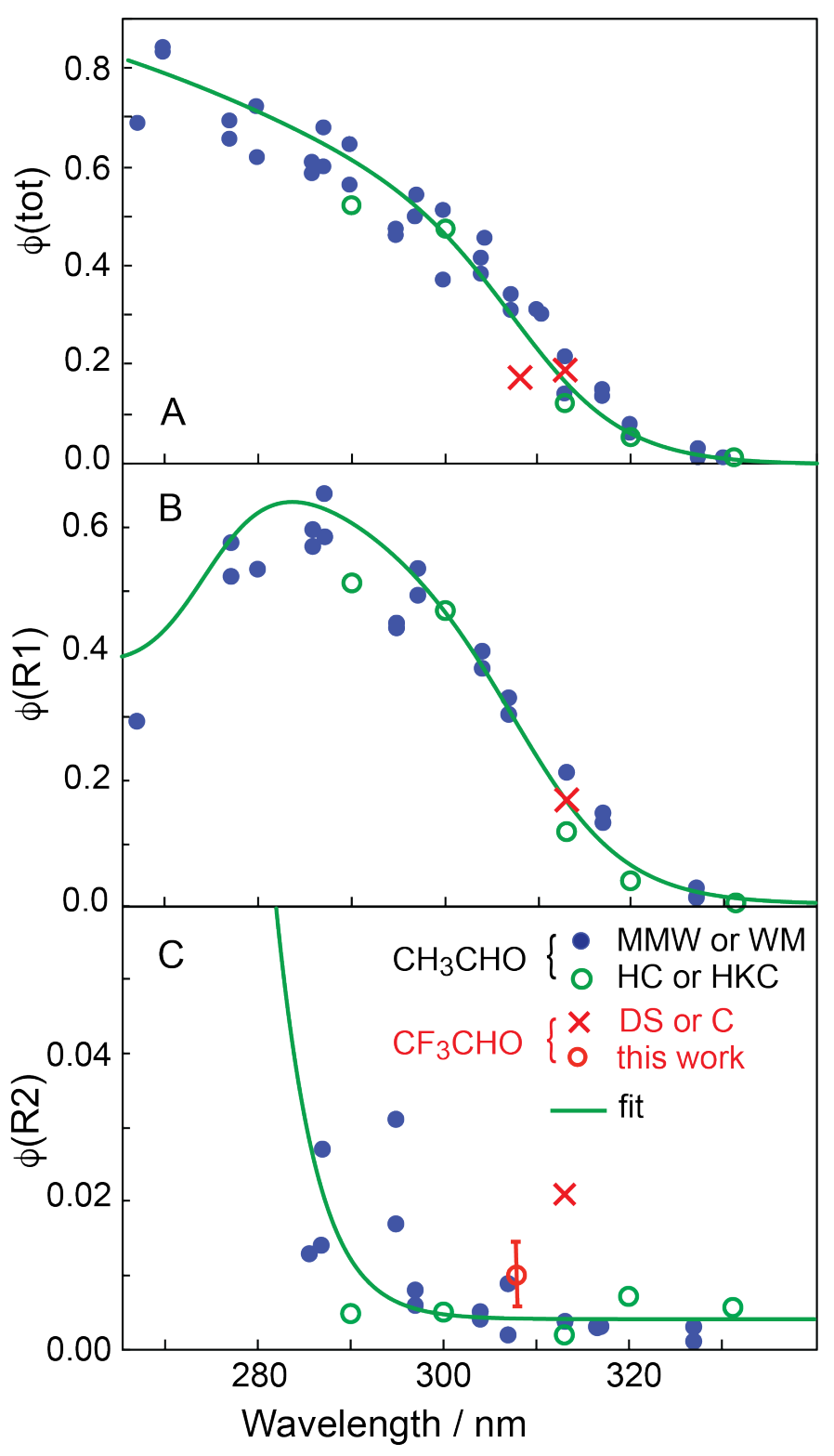

Figure 4: Comparison of quantum yields for $\mathrm{CH} 3 \mathrm{CHO}$ and $\mathrm{CF} 3 \mathrm{CHO}$ in 1 atm air, from various sources. [MMW: Reference [13]; WM: Reference [14]; HC: Reference [11]; HKC: Reference [12]. A) Total QY; B) QY for R1; C) QY for R2.

\section{Methods}

\section{Experimental}

In this work, we employed three experimental techniques to examine the three photochemical pathways, R1 to R3. Vacuum ultraviolet ionisation coupled with velocity-mapped ion imaging was used to investigate the $\mathrm{CF}_{3}$ and $\mathrm{CHO}$ radical fragments from R1. Resonance-enhanced multiphoton ionization with VMI was used to probe CO photoproducts from R2. FT-IR spectroscopy was used to probe the HF products from R3.

The apparatus for all experiments has been described in detail previously $[29,30,31]$ and the specific 
details (including preparation of the target compound by a literature method [32]) are in Supplementary Information (Supplementary Methods section Figure S1). In brief, a molecular beam of $2 \% \mathrm{CF}_{3} \mathrm{CHO}_{\text {or }}$ $\mathrm{CH}_{3} \mathrm{CHO}$ in helium was passed into a VMI spectrometer. The early time component of this beam was intersected by a photolysis laser $(308 \mathrm{~nm}$ ), followed by an ionisation (detection) laser. Radical fragments $\left(\mathrm{CF}_{3} / \mathrm{CH}_{3}\right.$ and $\left.\mathrm{CHO}\right)$ from $\mathrm{R} 1$ were probed at $115 \mathrm{~nm}$ to ionise all quantum states of the radical of interest. $\mathrm{CO}$ from R2 was detected in specific quantum states at $\sim 230 \mathrm{~nm}$ via REMPI. Nascent ions were separated in a time-of-flight mass spectrometer before impacting a position-sensitive $\mathrm{MCP} /$ phosphor screen detector, from which a kinetic energy distribution was determined. This is a collision free method that interrogates the unimolecular photochemistry of isolated molecules, providing true zero-pressure measurements. FT-IR experiments were conducted in a standard gas cell identifying end products following in situ photolysis at $308 \mathrm{~nm}$.

\section{Calculations}

The geometry optimisations and energy calculations were carried out at the G4(MP2)-6x level of theory [28] using the Gaussian 09 (Revision D.01) software package [33]. Results were visualised using the open-source Avogadro molecular editor.

\section{Methods References}

[29] N. Hobday, M. S. Quinn, K. Nauta, D. U. Andrews, M. J. T. Jordan, and S. H. Kable. Experimentaland Theoretical Investigation of Triple Fragmentation in the Photodissociation Dynamics of H2CO. Journal of Physical Chemistry A, 117(46):12091-12103, 2013.

[30] R. A. Ingle, C. S. Hansen, E. Elsdon, M. Bain, S. J. King, J. W. L. Lee, M. Brouard, C. Vallance,R. Turchetta, and M. N. R. Ashfold. Ultraviolet photochemistry of 2-bromothiophene explored usinguniversal ionization detection and multi-mass velocity-map imaging with a PImMS2 sensor. Journal of Chemical Physics, 147(1), 2017.

[31] A. E. Clubb, M. J. T. Jordan, S. H. Kable, D. L. Osborn, Phototautomerization of Acetaldehyde to Vinyl Alcohol: A Primary Process in UV-Irradiated Acetaldehyde from 295 to $335 \mathrm{~nm}$. Journal of Physical Chemistry Letters 3, 3522-3526 (2012).

[32] M. V. R. Reddy, M. T. Rudd, and P. V. Ramachandran. Study of fluorocarbonyls for the BaylisHillmanreaction. Journal of Organic Chemistry, 67(15):5382-5385, 2002.

[33] M. J. Frisch, G. W. Trucks, H. B. Schlegel, G. E. Scuseria, M. A. Robb, J. R. Cheeseman, G. Scalmani,V. Barone, B. Mennucci, G. A. Petersson, H. Nakatsuji, M. Caricato, X. Li, H. P. Hratchian, A. F. Iz-maylov, J. Bloino, G. Zheng, J. L. Sonnenberg, M. Hada, M. Ehara, K. Toyota, R. Fukuda, J. Hasegawa,M. Ishida, T. Nakajima, Y. Honda, O. Kitao, H. Nakai, T. Vreven, J. A. Montgomery, Jr., J. E. Peralta,F. Ogliaro, M. Bearpark, J. J. Heyd, E. Brothers, K. N. Kudin, V. N. Staroverov, R. Kobayashi, J. Nor-mand, K. Raghavachari, A. Rendell, J. C. Burant, S. S. Iyengar, J. Tomasi, M. Cossi, N. Rega, J. M.Millam, M. Klene, J. E. Knox, J. B. Cross, V. Bakken, C. Adamo, J. Jaramillo, R. Gomperts, R. E.Stratmann, O. Yazyev, A. J. Austin, R. Cammi, C. Pomelli, J. W. Ochterski, R. L. Martin, K. Mo-rokuma, V. G. Zakrzewski, G. A. Voth, P. Salvador, J. J. Dannenberg, S. Dapprich, A. D. Daniels, O.Farkas, J. B. Foresman, J. V. Ortiz, J. Cioslowski, and D. J. Fox. Gaussian09 Revision D.01. Gaussian Inc. Wallingford CT 2009.

\section{Acknowledgements}

The authors are grateful to Dr. David Osborn (Combustion Research Facility, Sandia National Laboratories) for insightful conversations in the early stages of this project; Prof. Timothy Schmidt for valuable conver- 
sations and advice throughout; Dr. Jonathon Ryan for advice and training on preparing $\mathrm{CF}_{3} \mathrm{CHO}_{\text {from }}$ its commercially available hydrate; Lorrie Jacob, Blair Welsh and Alain Lee for assistance with the FT-IR experiments and analysis. The support of the Faculty of Science Mechanical Workshop, particularly from James Richards, was critical to the outcomes of this work. This research includes computations using the computational cluster Katana supported by Research Technology Services at UNSW Sydney. This work was funded by the Australian Research Council (DE200100549/DP190102013). J.S.C. is supported by a UNSW Scientia PhD Scholarship. C.S.H. is supported by an Australian Research Council Discovery Early Career Researcher Award (DE200100549).

\section{Author contributions}

C.S.H. conceived the project. J.S.C. and C.S.H. designed and performed the experiments. All authors analysed and interpreted the results and contributed to the preparation of this manuscript.

\section{Competing interest declaration}

Authors declare no competing interests.

\section{Additional information}

Supplementary information is available for this paper at DOI:XXXX Correspondence and requests for materials should be addressed to C.S.H. 


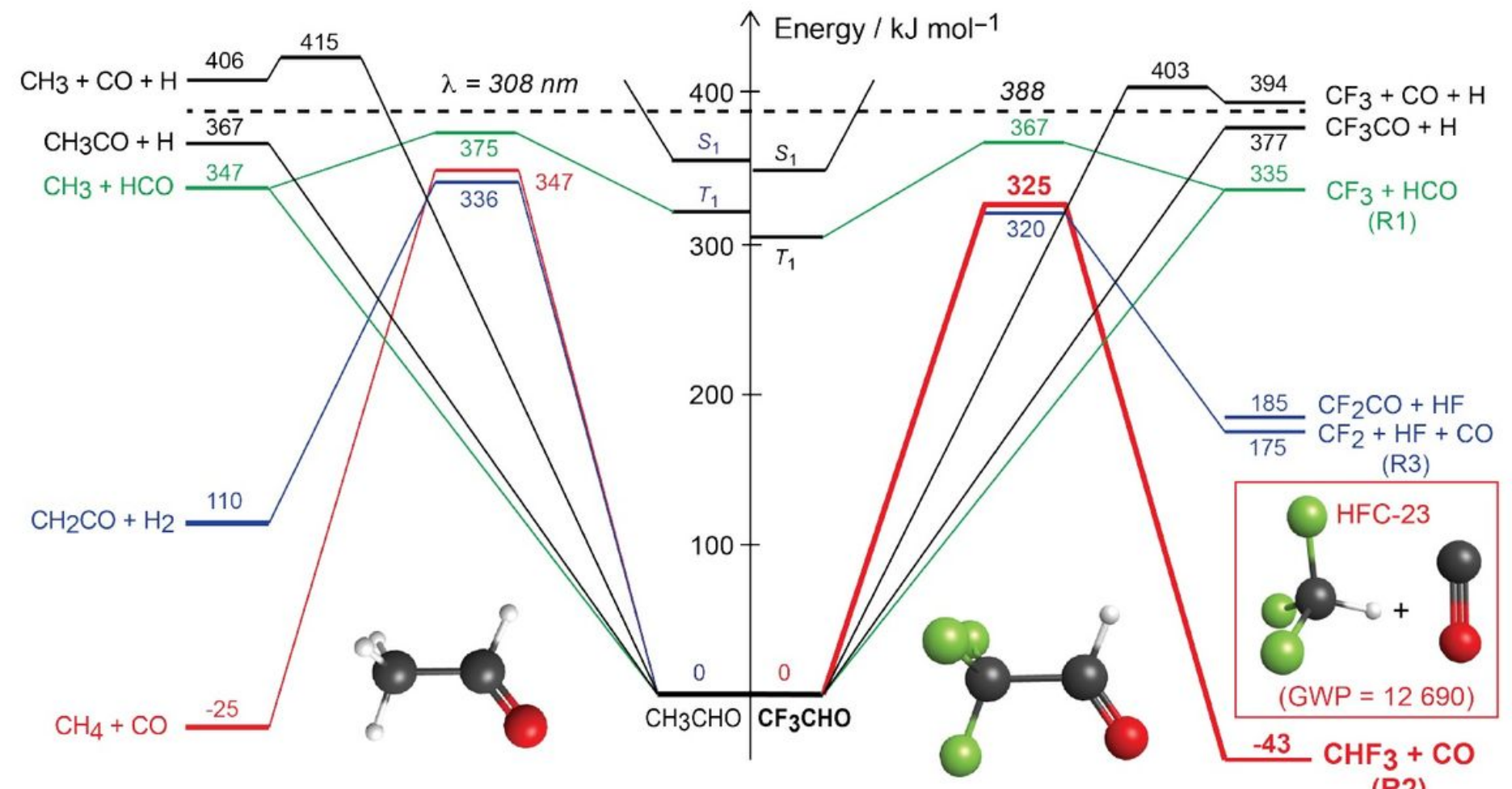

(R2)

\section{Figure 1}

Potential energy scheme for the photodissociation of $\mathrm{CF} 3 \mathrm{CHO}$ and $\mathrm{CH} 3 \mathrm{CHO}$. The $\mathrm{CH} 3 \mathrm{CHO}$ energies are experimental values from the references cited in the text and the $\mathrm{CF} 3 \mathrm{CHO}$ energies were calculated using the G4(MP2)-6x method [28]. The product and transition state energies are relative to the ground-state energy of the equilibrium geometry of each respective species. The dashed horizontal line represents the energy of a photon at $308 \mathrm{~nm}$. 


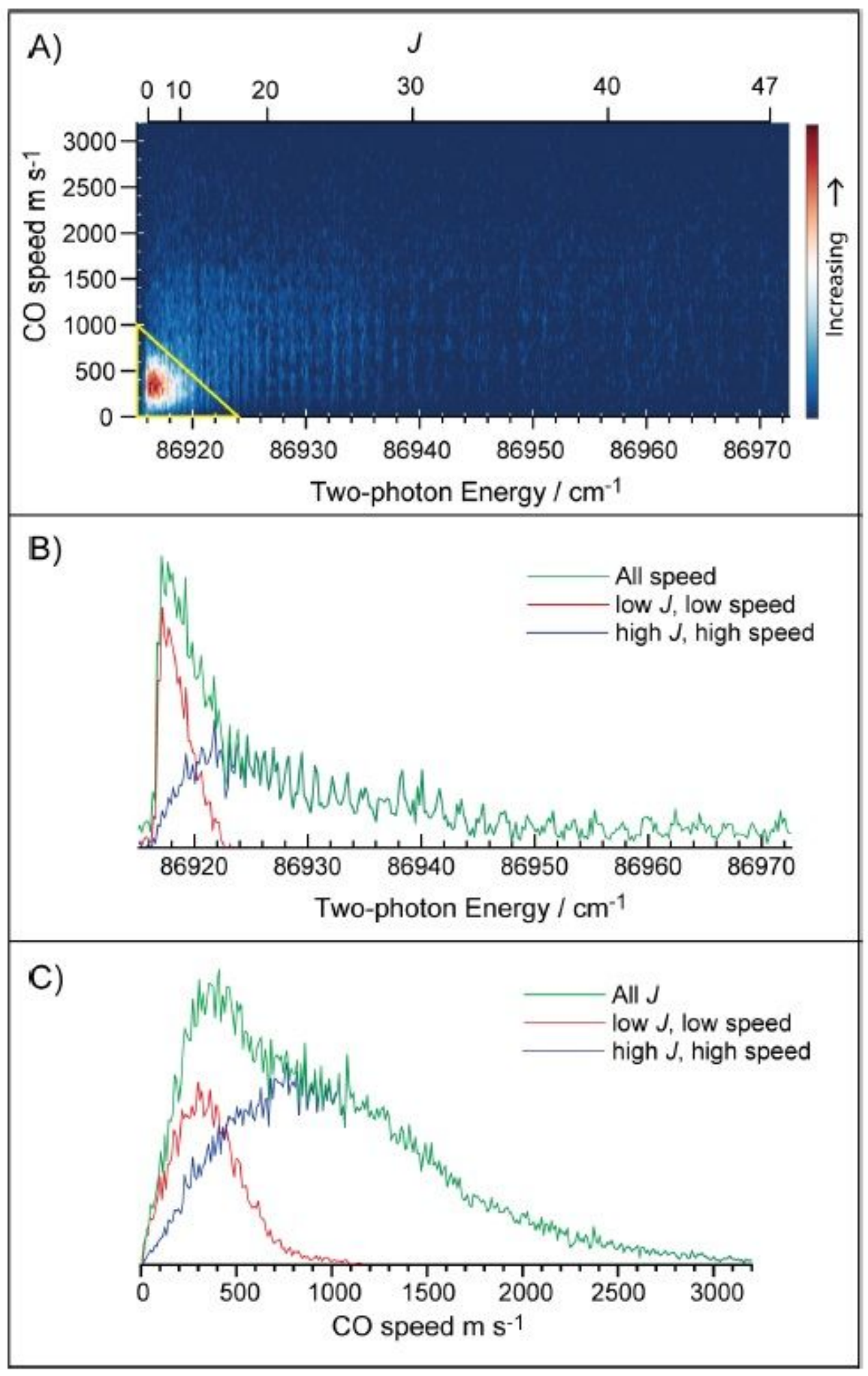

Figure 2

(A) 2D REMPI spectrum of the $\mathrm{CO}$ fragments formed following photolysis of $\mathrm{CF} 3 \mathrm{CHO}$ at $308 \mathrm{~nm}$. B) CO REMPI spectra from integrating (A) at low speed (within the yellow triangle, red), high speed (outside the yellow triangle, blue) and all speeds (green). C) Speed distributions determined by integrating (A) within and without the yellow triangle and across all $\mathrm{J}$. 


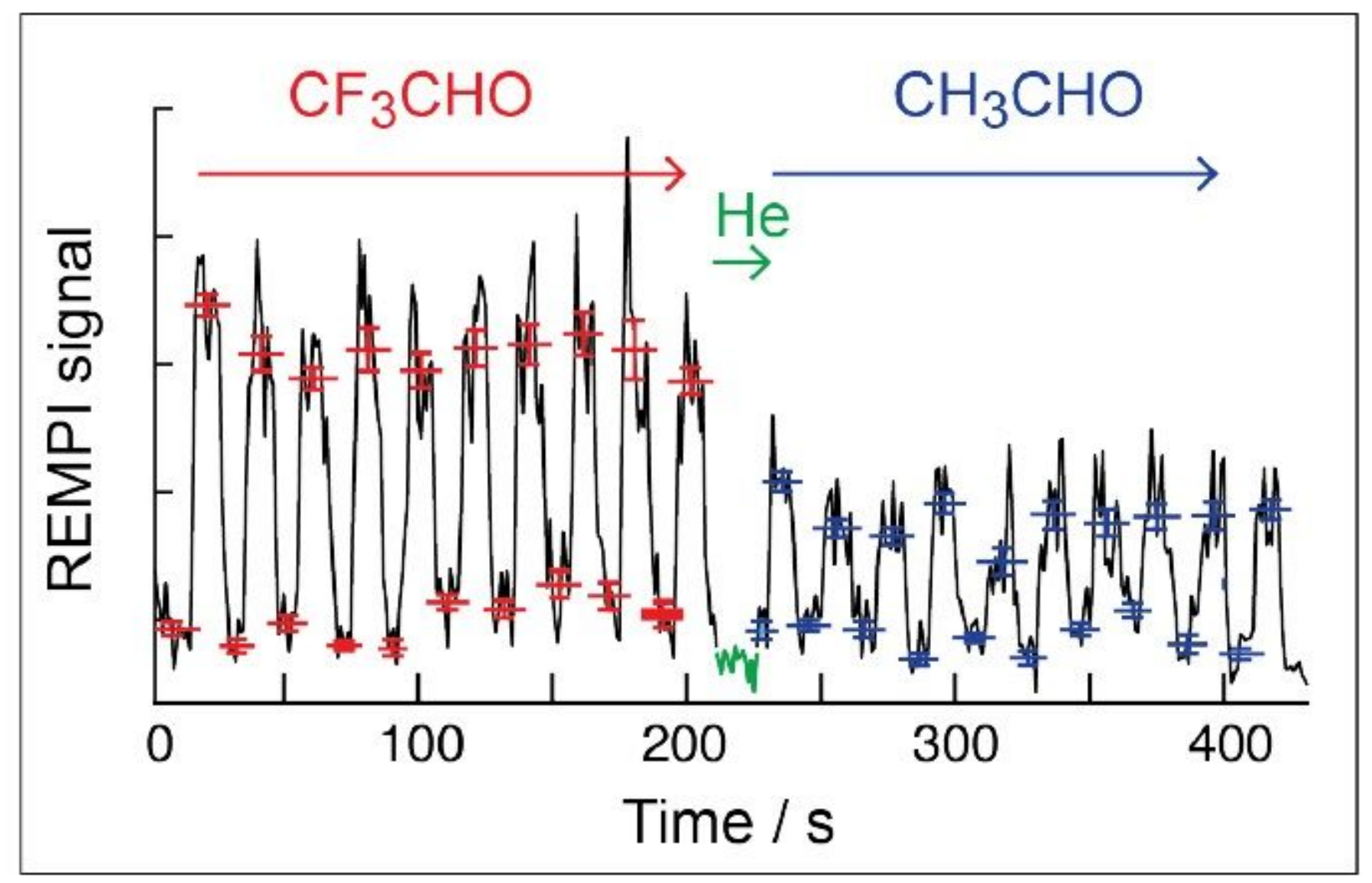

Figure 3

Time-dependent $\mathrm{CO}(\mathrm{J}=8)$ ion signal as the molecular beam is switched on and off and from CF3CHO to $\mathrm{CH} 3 \mathrm{CHO}$ as described in the text. 


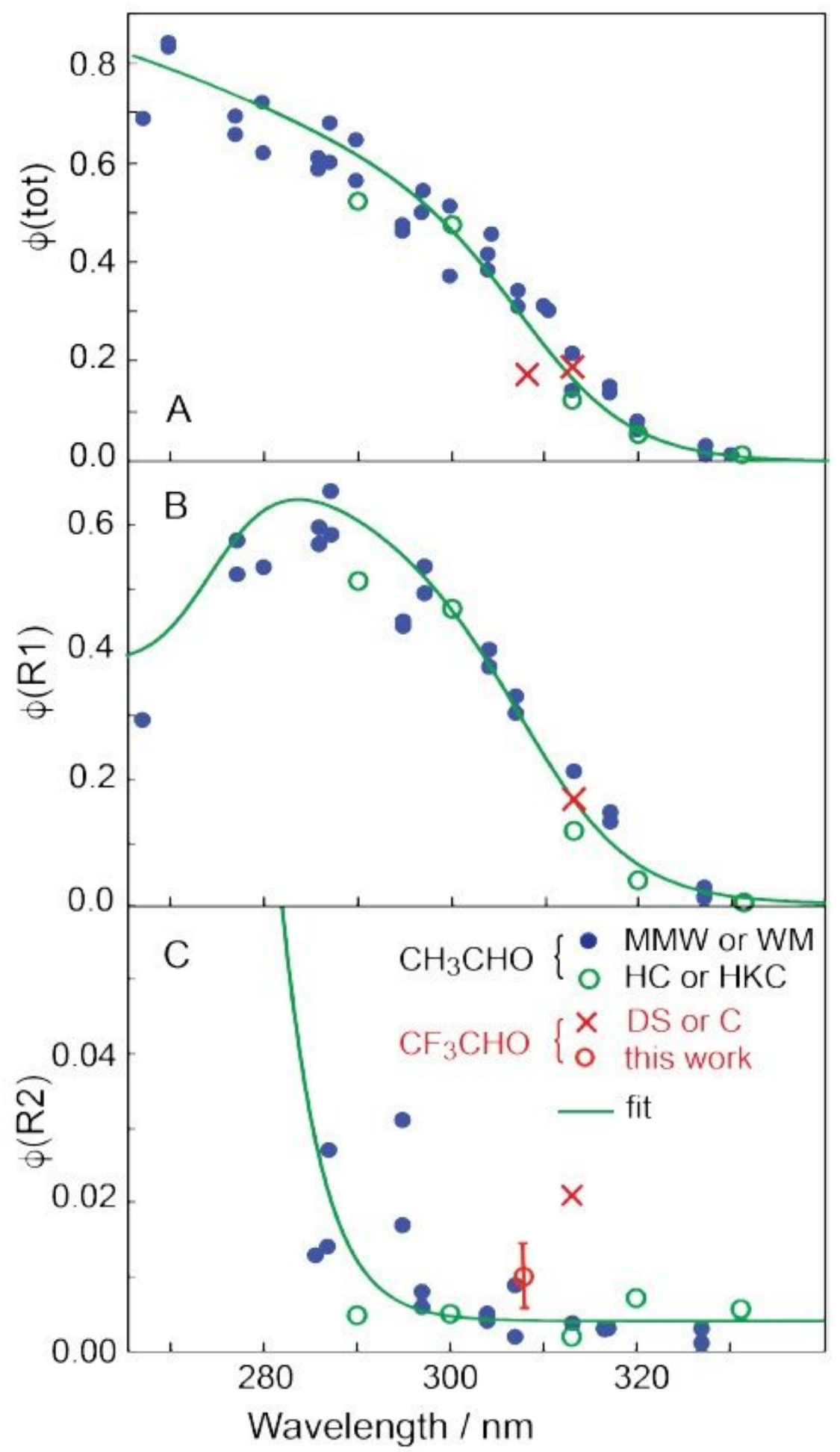

Figure 4

Comparison of quantum yields for $\mathrm{CH} 3 \mathrm{CHO}$ and $\mathrm{CF} 3 \mathrm{CHO}$ in 1 atm air, from various sources. [MMW: Reference [13]; WM: Reference [14]; HC: Reference [11]; HKC: Reference [12]. A) Total QY; B) QY for R1; C) QY for R2.

\section{Supplementary Files}


This is a list of supplementary files associated with this preprint. Click to download.

- CF3CHONaturesupp.pdf 